\title{
A look at important issues regarding safe orthokeratology ${ }^{+}$
}

\author{
E Chetty ${ }^{*}, \mathrm{~S}$ Jackson ${ }^{*}, \mathrm{C}$ Mitton ${ }^{*}$ and TK Phillips \\ Department of Optometry, University of Johannesburg, PO Box 524, Auckland Park, 2006 South \\ Africa
}

< stevejacksonster@gmail.com >

The prospect of getting through the day without the inconvenience of spectacles or contact lenses would be a utopia for any myope. Concerns of fogged up spectacles or dried out contact lenses in the air-conditioned office would be a thing of the past. But at what price does this pseudo-emmetropic lifestyle come at? The incidence of myopia varies across the world although Asian countries appear to be experiencing an epidemic of the condition. A report ${ }^{1}$ from Japan and Taiwan showed a $50 \%$ overall incidence and an $84 \%$ incidence in people under the age of 16 years respectively whereas the incidence of myopia in Europe and America was 20\%-30\% and $10 \%-20 \%$ in Africa ${ }^{2}$. Early education being an epidemiological parameter is only one of many predisposing factors that contribute to the development of myopia. Other factors include genetic predisposition, axial length of the eye and corneal curvature, to name a few ${ }^{2,3}$.

We currently live in an era that revolves around seeking comfort in all aspects of life from doing your banking in your lounge to having an all-in-one camera, radio, email and phone. Therefore, it is not surprising that there is so much effort invested in making the management of refractive errors more convenient and thus conventional spectacles and contact lenses will no longer do. In its vastness, modern technology has provided methods that can purge refractive error such as laser refractive surgery. Such methods are not available to everybody and also carry their own risks.

Orthokeratology, which was a serendipitous discovery, is another procedure used to manage myopia. Orthokeratology reduces myopia and provides adequate unaided vision for an average of an eight-hour working day $^{4}$, by a process that is still not entirely understood. Essentially, specifically designed rigid gas permeable lenses are used to thin out the central cornea thereby decreasing the refractive error temporarily ${ }^{5}$. This procedure is most effective in patients with low to moderate myopia with low astigmatism ${ }^{4}$. Studies have reflected similar results with reductions in myopia averaging between $1.75 \mathrm{D}-3.33 \mathrm{D}$ with many patients achieving good unaided visual acuity ${ }^{6-9}$.

The efforts made to reduce or eliminate myopia date as far back as the 1950's with the development of polymethyl methacrylate (PMMA) contact lenses ${ }^{9}$. Clinicians noticed that PMMA corneal contact lenses that were fitted flat inadvertently altered corneal shape and refractive error and in some patients, the flat fitting lenses had stopped myopic progression for up to two years ${ }^{9}$. This observation gave rise to the interest in the possibility of designing a contact lens that could flatten the cornea permanently and eradicate myopia. The idealistic goal lead to many clinical investigations starting in the 1960's beginning with the Orthofocus technique ${ }^{9}$, and continuing until current day orthokeratology.

Using the basic concept of flattening the cornea to decrease myopia, George Jessen developed the Orthofocus technique ${ }^{9}$. His technique included the use of a plano-powered PMMA contact lens and the degree

\footnotetext{
${ }^{+}$This review is based on work performed as part of the requirements for the course Contact Lenses B under the supervision of Professor WDH Gillan at the University of Johannesburg

* Fourth-year students registered for the degree BOptom

Received 21 August 2007; revised version accepted 30 October 2007
} 
of the flat fit was determined by the patient's myopia. The myopia was corrected by the tear lens formed behind the contact lens. With continued use, the flattening of the cornea provided satisfactory unaided vision after lens removal.

Since Jessen's initial success in reducing myopia, many other practitioners continued trials to improve orthokeratology. The general technique involved the use of large diameter PMMA lenses that were fitted flatter than $\mathrm{K}$ and used during the day to provide reasonable unaided vision for the afternoon and evening. The hype lasted 20 years during which many general conclusions were derived. One of these conclusions was that the flat-fitting PMMA lenses did reduce myopia, but by an amount that was not much more than that which was reduced with conventional PMMA contact lenses fitted on alignment ${ }^{9}$. Also, the reduction was not only temporary but unpredictable and variable. Refractive error and corneal shape eventually returned to the original state after lens wear was stopped. The flat fit of the lens resulted in decentration and instability of the lens on the cornea. It also resulted in irregular corneal astigmatism and regular with-the-rule astigmatism ${ }^{4,9,10}$. Due to limited technology at that time, further development of orthokeratology slowed down.

Along with the late 1980's came a revolutionary benchmark for healthier contact lens fitting. The introduction of the rigid gas permeable (RGP) lenses with their high oxygen permeability allowed for the extended wear of contact lenses ${ }^{9}$. Other new developments that sparked renewed interest in orthokeratology in the 1990's were the accessibility of corneal topographers and, more importantly, the design of reverse geometry lenses.

Hypoxia has always been a fundamental health issue with the fitting of contact lenses. In the normal eye that is free of any unnatural barrier such as contact lenses, $4 \%-6 \%$ corneal swelling is induced by closed eye conditions such as sleep ${ }^{11}$. Upon waking, this minimal corneal oedema dissipates gradually with no clinical consequence. PMMA lenses are completely impermeable to oxygen and therefore wearing them during open eye conditions still posed health risks to the integrity of the cornea. Practitioners attempted to overcome corneal hypoxia by making the contact lens diameter as small as possible. Some also resorted to making fenestrations in the lens ${ }^{11}$. During the 1970 's the first gas permeable contact lens became available but it also had its constraints, such as susceptibility to protein deposits and low Dk values. It was only in the 1980's that gas permeable materials improved greatly, having permeability above 20-30 Dk units and more resistance to protein deposits ${ }^{11}$. It is this Fluoro-Silicone/Arcrylate material that made extended wear of rigid lenses possible thus leading to overnight orthokeratology 9,11 .

Currently there are numerous rigid gas permeable materials on the market that range between $60-100 \mathrm{Dk}$ and some even higher than this. Choice of material is dependent on what the material is going to be used for, for example, daily wear contact lenses or extended wear contact lenses. Holden and Mertz ${ }^{12}$ found that for the safe use of a contact lens overnight, a Dk/t value of $\left.87.0 \times 10^{9}\left(\mathrm{~cm} \mathrm{x} \mathrm{mLO}_{2}\right) / \mathrm{sec} \times \mathrm{ml} \times \mathrm{mmHg}\right)$ and an equivalent oxygen percentage (EOP) of $18 \%$ was required to confine overnight swelling to a normal amount of $4 \%$. The permeability of gas permeable materials used in current orthokeratology lenses meet and sometimes even exceed this minimum $\mathrm{Dk} / \mathrm{t} .{ }^{4}$ The overnight orthokeratology procedure requires that the patient sleeps with the gas permeable contact lens and upon waking the patient removes the contact lens and should be provided with clear unaided vision for the day. Removal of the contact lens in the morning is the differentiating factor between orthokeratology lenses and extended wear lenses. With orthokeratology, the lens is removed every morning thus providing the whole day for the cornea to 'breath' and recover from the overnight corneal oedema caused by the diminished oxygen availability. Nichols et $a 1^{4}$ reported the absence of neovascularization and corneal oedema which indicates that there is no clinically significant hypoxia induced by overnight wear of orthokeratology lenses. Other studies have also deemed overnight orthokeratology safe with respect to hypoxia ${ }^{6,10}$ provided that patients comply with safety protocols. However, these studies have been conducted over short trial periods therefore more investigation is required to look into the long term effects of orthokeratology on corneal oedema. Conversely, some other studies have found significant corneal swelling ${ }^{13}$ as well as superficial corneal staining that may have been induced by hypoxia ${ }^{6}$. These adverse effects were found immediately after lens removal and dissipated along the course of the day. The contradictory results found in these studies emphasize the need for 
more research into the hypoxia induced by orthokeratology lenses and its long term effects on corneal health. Although hypoxia does not seem to pose a major problem for now, there are other corneal health risks that patients are exposed to due to overnight orthokeratology.

Currently, orthokeratology employs the use of reverse geometry lenses. This lens design allows for predictable results and has eliminated problems of lens instability and decentration, issues that were encountered by early orthokeratology lenses. Although there are many different reverse geometry lens designs on the market, they are all designed based on the same general principle. Studies have been done to compare the efficacy of the different designs and all of them arrived at similar conclusions. To mention a couple, Soni et al and Tahhan et $a l^{10}$ both concluded that there was no clinically significant difference in the performance of each of the different lens designs used in their studies. As suggested by Soni $e l^{2} l^{7}$, the reason for achieving similar results is that all lens designs are fundamentally the same.

A conventional rigid lens is designed such that the central base curve is surrounded by concentric curves that flatten out progressively toward the periphery. With the reverse geometry lens design, the central optic zone is fitted flatter than the central corneal curvature. Steeper secondary curves surround the central optic zone. A flat central base curve is possible because these secondary steep or reverse curves provide the lens with stability and aids in centering the lens as well. Beyond the reverse curves are flatter curves that are fitted on alignment. The weight of the lens is supported in the periphery by these peripheral alignment curves. A well-fitted orthokeratology lens would have a fluorescein pattern that indicates corneal clearance under the steep curve zone and corneal alignment of the optic zone ${ }^{9}$. The pooling that is seen under the steep curve zone is due to a tear reservoir that is created between the cornea and the lens ${ }^{4}$. This tear reservoir facilitates the health of the cornea by increasing oxygen availability to the cornea.

When fitting one's patients with contact lenses, some of the most important considerations of the practitioner are the patient's safety and ocular health. These considerations are no different when performing orthokeratology on patients, and arguably even more important in this procedure in which unnatural corneal reshaping is taking place. Therefore, it is important to consider the issues when it comes to orthokeratology associated corneal infections and ulcers.

With the increase in popularity of orthokeratology in the past decade, and growing evidence for the better efficacy of the modern procedures ${ }^{9}$, there has also been an increased incidence of orthokeratology associated microbial keratitis. This should worry any practitioner who plans to perform orthokeratology and who cares about the ocular health of patients. No matter how small the incidence of these infections are, for those patients who are infected, it is a vision threatening event and it is the responsibility of the practitioner.

From what has been reported in the literature in the past few years, the most common causes of microbial keratitis related to orthokeratology have been Pseudomonas aeruginosa ${ }^{14-17}$, Acanthamoeba ${ }^{14-16,18}$ as well as pathogens such as Pseudomonas putida, Burkholderia cepacia ${ }^{19}$, Nocardia asteroids, Providencia stuartii, fungus ${ }^{16}$, and Serratia marcescens ${ }^{20}$.

Orthokeratology has become increasingly popular in Asia over the past decade due to the high prevalence of myopia in these countries ${ }^{8}$, and it is therefore no surprise that many of the reports dealing with complications of orthokeratology have been coming out of countries like China, Taiwan and Hong Kong. However, infections related to orthokeratology are also occurring in other countries such as Canada and Australia. According to Watt et $\mathrm{l}^{15}$, between the years 2000 and 2007, there have been over 100 cases of microbial keratitis reported in international literature. Watt and Swarbrick ${ }^{14}$ reviewed the first 50 of these cases and reported that $80 \%$ of them were from East Asia, and $61 \%$ of the 50 cases were in children. Pseudomonas aeruginosa caused $52 \%$ of the infections while Acanthamoeba caused $30 \%$ of them.

When comparing reported cases of microbial keratitis from different countries, there is a similar pattern of infections. In the Beijing Institute of Ophthalmology (China) between March 2000 and August 2001, 28 cases of microbial keratitis related to overnight orthokeratology were diagnosed ${ }^{16} .75 \%$ of these infections were caused by Pseudomonas aeruginosa while Acanthamoeba caused the majority of the rest of the infections. More recently, other cases of Acanthamoeba infections have been reported in China ${ }^{18}$. In one survey conducted in 
Australia, it was found that between 1997 and 2005 there were nine reported cases of microbial keratitis associated with orthokeratology ${ }^{15}$. In six of these cases, the infecting organisms were identified. Four were caused by Pseudomonas aeruginosa and two by Acanthamoeba.

Other organisms besides Pseudomonas aeruginosa and Acanthamoeba such as Serratia marcescens have also been reported ${ }^{20}$ to have caused microbial keratitis associated with orthokeratology. However, Pseudomonas aeruginosa and Acanthamoeba appear to be the main cause of infection at this point in time. It is no coincidence that the two most common microorganisms causing standard contact lens microbial keratitis are also Pseudomonas aeruginosa and Acanthamoeba. At the end of the day, orthokeratology is a form of contact lens wear. Keeping this in mind, perhaps one is then able to look at what happens when there is infection related to normal contact lens wear and compare it to orthokeratology, and then try to find ways to make orthokeratology a safer form of contact lens wear.

Some of the general risk factors for infectious keratitis related to contact lens wear include the alteration of normal ocular flora, noncompliance when it comes to disinfection and storage, biofilm formations, altered ocular defenses such as altered tears and epithelium, and hypoxia ${ }^{11}$. There is a wide range of organisms that make up the ocular flora, and a disturbance in the natural balance of this flora increases the risk of corneal infection $^{11}$. In a longitudinal study of eye flora over a period of a few months after the fitting of orthokeratology lenses, Boost and $\mathrm{Cho}^{21}$ concluded that there are little or no alterations in ocular flora by orthokeratology lens wear over a long period of time, and that the presence of any contaminants are only transient. This rules out a possibility that a change in ocular flora is a major cause of microbial keratitis.

Noncompliance by patients when it comes to lens disinfection and storage is probably one of the main causes of corneal infections related to contact lenses and there is much literature which shows noncompliance to be a major problem in contact lens practice ${ }^{11}$. This is also one of the driving factors for the development of simple one step contact lens disinfecting and storage solutions. The literature dealing with orthokeratology related infections strongly suggests that there is poor patient compliance with orthokeratology lenses ${ }^{14-16,18,19,21}$ including poor lens care, washing lenses with tap water, irregular disinfection and infrequent replacement of lens cases (which are major causes of the build up of biofilms in lens cases ${ }^{21}$ ), storing lenses in saline and noncompliance with follow up.

Another general risk factor for contact lens related microbial keratitis is the altering of ocular defenses such as altered tear film, altered epithelium and trauma ${ }^{11}$. Tears have a number of antibacterial properties and a change in tear composition and flow may increase the risk of an infection. This should be kept in mind with orthokeratology lenses that are worn while sleeping where there is reduced tear flow and reduced washing away of pathogens by blinking ${ }^{21}$, as well as all the other effects of overnight contact lens wear. One such proposed effect is that the closed eyelids lead to higher temperatures and help incubate bacteria under the lenses ${ }^{16}$. It is unlikely, however, that these factors alone are the main causes of infections, as infectious keratitis has also been reported in daytime orthokeratology where the tear flow is more normal and there is blinking ${ }^{19}$. Thus a combination of factors must be considered when trying to pin point a cause for an infection.

Altered epithelium and trauma are other risk factors for contact lens related microbial keratitis that may have a great significance in orthokeratology related infections. Normal corneal epithelium acts as a barrier to almost all bacteria, thus serving as a major protective structure to the cornea. Any defect or trauma to the epithelium increases the risk of infection. The reverse geometry contact lenses that are used today to perform orthokeratology have a flat central curvature, which puts pressure on the cornea, and peripheral curves steeper than the central curves. Firstly, it is obvious that a poorly fitted orthokeratology lens can be traumatic to the epithelium and lead to infection. However, even if the lens is 'correctly' fitted, it has been shown that reverse geometry lenses cause central epithelial thinning and redistribution in response to tear film forces created behind the reverse geometry lenses ${ }^{5,22}$, and it is possible that this thinning also affects the epithelial barrier function. More research needs to be conducted to confirm this. It has, however, been shown that regular rigid gas permeable extended wear causes a reduction in the epithelial barrier function and that the permeability of the epithelium increased significantly after overnight wear of rigid gas permeable lenses, even from high-Dk 
contact lenses $^{23}$. This is as a result of a combination of hypoxia of the epithelium, the mechanical stress of the presence of the contact lens and eye closure. If it is true that a normal rigid gas permeable contact lens which is not designed to reshape the cornea is able to impair the epithelial barrier function, then it is likely that reverse geometry lenses impair this barrier to a greater extent, because there is a much greater mechanical stress placed on the epithelium during eye closure, as well as all the regular effects of a rigid gas permeable lens on the eye. It has also been shown that there is epithelial hypoxia and corneal swelling as soon as reverse geometry lenses (with a Dk of 100) are removed in the morning ${ }^{13}$. From all this it is possible to conclude that orthokeratology lenses alter the epithelium to a large extent, and cause a transient state of hypoxia. These factors may be the prime causes of microbial keratitis due to an impaired epithelial barrier function.

After examining the general risk factors for infectious keratitis related to contact lens wear and reverse geometry lenses, it becomes clearer why the two main pathogens involved in infectious keratitis associated with orthokeratology are Pseudomonas aeruginosa and Acanthamoeba. Pseudomonas aeruginosa is a gram-negative bacterium, which is an opportunistic pathogen, and requires epithelial damage to infect the cornea ${ }^{15}$. It does not normally adhere to a healthy cornea because of the protective mucous layer of the tears as well as the glycocalyx of the epithelial cell surface ${ }^{24}$. Contact lens wear depletes these protective layers and thus increases the chance of Pseudomonas aeruginosa infecting the cornea. It is then perhaps clearer why this pathogen is the prime cause of orthokeratology related lens wear infections. As already described above, factors such as noncompliance, trauma, altered epithelium and tears from the use of reverse geometry lenses would all contribute to these infections.

Acanthamoeba is a free living amoeba which is found all over the natural environment such as in soil, dust, water, air, and even in the nasopharyngeal mucosa of healthy humans ${ }^{24}$. Acanthamoeba infections are rare in extended rigid contact lens wear, and infections have been linked to the exposure of contact lenses to contaminated water ${ }^{15}$. It is therefore thought that the high rate of Acanthamoeba infections seen in orthokeratology patients is due to exposure of the lenses to contaminated sources such as tap water, saline solutions and contaminated storage solutions. This is supported by the literature in which case histories reveal a large percentage of noncompliance ${ }^{14-16,18,19,21}$.

So what does this all mean for a practitioner who wants to practice safer orthokeratology? Firstly, one would have to fit the reverse geometry lenses properly and with the best materials available, which would decrease the chances of damaging the epithelium through trauma and hypoxia. This means constantly keeping up with the literature dealing with orthokeratology and contact lenses. Secondly, educating ones patients properly about how to insert and remove lenses in a safe manner, as well as teaching proper lens care. This includes frequent disinfections, regular replacement of lens cases, not using tap water, saline and old solutions for any steps in lens care, and air-drying lenses ${ }^{14-16}$. The practitioner should constantly reinforce the importance for continued compliance. It is also important for the patient to understand the importance of follow up appointments, as well as the common signs and symptoms of corneal infections so that if one does occur, it can be attended to as quickly as possible. All these safety measures are especially important when patients are children, and in these cases parents should be educated as diligently as the patients ${ }^{8,21}$. Boost and Cho ${ }^{21}$ pointed out, "The public/press are less forgiving when the vision of a child is adversely affected by lens wear, even if the likely cause was noncompliance of the patients." Constant reinforcement and monitoring of children is essential if this procedure is to work.

Other issues that are also important to look at in orthokeratology are the various physical changes that occur in the cornea. It has already been mentioned that research has shown that reverse geometry lenses cause the corneal epithelium to be redistributed and molded, causing central corneal thinning as well as midperipheral stromal thickening ${ }^{4}, 5,13,22,25$. Little research has been performed to gain an exact understanding of what is happening to the cornea on a histological level during orthokeratology and the exact cellular processes occurring, clinical significances, and pathophysiological ramifications are still poorly understood. Swarbrick et $a l^{5}$ concluded that any procedure that causes such dramatic changes in corneal thickness must be viewed with caution. So, with all the advances we have made concerning orthokeratology we must still keep in mind that 
what we are doing to the cornea is unnatural, and that we know very little about the long term effects of what we are doing to the physical state of the cornea.

Another physical change that can occur during orthokeratology is the flattening of the posterior cornea during the initial adaptation period of reverse geometry lenses ${ }^{26}$. Even though the posterior surface of the cornea contributes a small amount of the refractive power of the cornea, it is once again important to know of these unnatural physical changes orthokeratology causes.

Other less serious conditions that have been reported from orthokeratology include the formation of a faint brown or white-pigmented ring in the basal epithelium of the midperipheral part of the cornea ${ }^{27}$, central corneal staining ${ }^{28,29}$, epithelial microcysts ${ }^{6}$, and lens binding ${ }^{28,29}$. The pigmented ring is similar to Fleisher's ring seen in keratoconic eyes, and is believed to be an iron deposition as a result of rapid corneal curvature change and pooling of tears in that area due to the reverse geometry lenses ${ }^{27}$. A white lesion of unknown etiology in this type of pigmented ring has also been reported ${ }^{30}$. These pigmentations do not affect vision and do not appear to have any clinical significance.

Epithelial microcysts represent evidence of chronic metabolic stress and altered cellular growth patterns caused by the effects of hypoxia and hypercapnia ${ }^{31}$. Although studies differ on whether or not overnight orthokeratology lens wear causes clinically significant hypoxia, one study has shown that a significant amount of corneal oedema results from overnight wear of rigid gas permeable lenses with both normal rigid lenses and reverse geometry lenses ${ }^{13}$. This oedema indicates hypoxia and may explain the presence of epithelial microcysts reported as well as the superficial corneal staining present in most patients ${ }^{17}$. Lens binding is one of the complications associated with rigid gas permeable extended wear ${ }^{32}$. It is caused by the thinning of the posterior tear film during sleep due to lid pressure, which results in a mucous rich sticky tear film. This then causes the lens to bind to the cornea ${ }^{33}$. It has also been reported to occur as a result of overnight reverse geometry lens wear $^{28,29}$. It is important for the practitioner to teach the patient how to free a bound lens if it were to occur.

In conclusion, it is evident from the above discussions that the art of orthokeratology has come a long way. The major problem of hypoxia has been dealt with to a large extent with the arrival of gas permeable lens materials as well as new lens designs. There are, however, complications such as infectious keratitis that pose a major threat to the safe practice of orthokeratology. It is important for the practitioner to be aware of the possible causes of these infections, and educate one's patients about the risks of infections and diligent lens care systems. Certain physical changes occur in the cornea with orthokeratology and more research needs to be conducted to gain an understanding of the exact mechanisms behind this controversial procedure. This will inevitably bring about a greater understanding of the associated complications and this understanding will ultimately lead to safer orthokeratology practice.

\section{Acknowledgement}

To Professor WDH Gillan for his suggestions for improving this article.

\section{References}

1. Fan DSP, Lam DSC, Lam RF, Lau JTF, Chong KS, Cheung EYY, Lai RYK, Chew SJ. Prevalence, incidence, and progression of myopia of school children in Hong Kong. Investigative Ophthalmology and Visual Science 2004 45 1071-1075.

2. Fredrick DR. Myopia. British Medical Journal 2002324 1195-1199.

3. Zadnik K, Mutti DO, Friedman NE, Qualley PA, Jones LA, Qiu P, Hsu JC, Moeschbergur ML. Ocular predictors of the onset of juvenile myopia. Investigative Ophthalmology and Visual Science 199940 1936-1943.

4. Nichols JJ, Marsich MM, Nguyen MBS, Barr JT, Bullimore MA. Overnight orthokeratology. Optometry and Vision Science 200077 252-259.

5. Swarbrick HA, Wong G, O'Leary DJ. Corneal response to orthokeratology. Optometry and Vision Science 1998 75 791-799. 
6. Rah MJ, Jackson JM, Jones LA, Marsden HJ, Bailey MD, Joseph T. Overnight orthokeratology: preliminary results of the lenses and overnight orthokeratology (LOOK) study. Optometry and Vision Science 200279598 605.

7. Soni PS, Nguyen TT. Overnight orthokeratology experience with XO material. Eye and Contact Lens 200632 3945.

8. Kwok LS, Pierscionek BK, Bullimore M, Swarbrick HA, Mountford J, Sutton G. Orthokeratology for myopic children: wolf in sheep's clothing? Clinical and Experimental Ophthalmology 2005 33 343-347.

9. Swarbrick HA. Orthokeratology review and update. Clinical and Experimental Optometry 2006 89(3) 124-143.

10. Tahhan N, Du Toit R, Chung H, Hood D, Holden B. Comparison of reverse-geometry lens designs for overnight orthokeratology. Optometry and Vision Science $2003 \mathbf{8 0}$ 796-804.

11. Bennett ES, Weissman BA. Clinical contact lens practice. Philadelphia: Lippincott Williams and Wilkins, 2005.

12. Holden BA, Mertz GW. Critical oxygen levels to avoid corneal edema for daily and extended wear contact lenses. Investigative Ophthalmology and Visual Science 198425 1161-1167.

13. Wang J, Fonn D, Simpson TL, Sorbara L, Kort R, Jones L. Topographical thickness of the epithelium and total cornea after overnight wear of reverse-geometry rigid contact lenses for myopia reduction. Investigative Ophthalmology and Vision Science 200344 4742-4746.

14. Watt K, Swarbrick HA. Microbial keratitis in overnight orthokeratology: Review of the first 50 cases. Eye and Contact Lens 200531 201-208.

15. Watt K, Boneham GC, Swarbrick HA. Microbial keratitis in orthokeratology: the Australian experience. Clinical and Experimental Optometry 200790 182-189.

16. Sun X, Zhao H, Deng S, Zhang Y, Wang Z, Li R, Luo S, Jin X. Infectious keratitis related to orthokeratology. Ophthalmic Physiological Optics 200626 133-136.

17. Priel A, Grinbaum A, Barequet IS. Severe Pseudomonas aeruginosa keratitis shortly after initiation of corneal refractive therapy. Eye and Contact Lens 200632 1-2.

18. Sun X, Chen L, Zhang Y, Wang Z, Li R, Luo S, Jin X. Acanthamoeba keratitis as a complication of orthokeratology. American Journal of Ophthalmology 2003136 1159-1161.

19. Ying-Cheng L, Chao-Kung L, Wen-Ming H. Daytime orthokeratology associated with infectious keratitis by multiple gram-negative bacilli: Burkholderia cepacia, pseudomonas putida, and pseudomonas aeruginosa. Eye and Contact Lens 200632 19-20.

20. Ko-Hua C, Tung-Mei K, Wen-ming H. Serratia marcescens corneal ulcer as a complication of orthokeratology. American Journal of Ophthalmology 2001132 257-258.

21. Boost MV, Cho P. Microbial flora of tears of orthokeratology patients, and microbial contamination of contact lenses and contact lens accessories. Optometry and Vision Science 200582 451-458.

22. Sridharan R, Swarbrick HA. Corneal response to short-term orthokeratology lens wear. Optometry and Vision Science 200380 200-206.

23. Lin MC, Graham AD, Fusaro RE, Polse KA. Impact of rigid gas-permeable contact lens extended wear on corneal epithelial barrier function. Investigative Ophthalmology and Visual Science 200243 1019-1024.

24. Efron N. Contact lens-induced microbial infiltrative keratitis. Optician 1997 No 5617214

25. Alharbi A, Swarbrick HA. The effects of overnight orthokeratology lens wear on corneal thickness. Investigative Ophthalmology and Vision Science 200344 2518-2523.

26. Owens H, Garner LF, Craig JP, Gamble G. Posterior corneal changes with orthokeratology. Optometry and Vision Science 200481 421-426.

27. Cho P, Chui WS, Mountford J, Cheung SW. Corneal iron ring associated with orthokeratology lens wear. Optometry and Vision Science 200279 565-568.

28. Cho P, Cheung SW, Edwards MH, Fung J. An assessment of consecutively presenting orthokeratology patients in a Hong Kong based private practice. Clinical and Experimental Optometry 200386 331-338.

29. Chui WS, Cho P. Recurrent lens binding and central island formations in a fast-responding orthokeratology lens wearer. Optometry and Vision Science 200380 490-494.

30. Cheung SW, Cho P, Cheung A. White lesion in the corneal pigmented ring associated with orthokeratology. Ophthalmic Physiological Optics. 200525 264-268.

31. Efron N. Contact lens-induced epithelial microcysts. Optician 1996 No 5549211. 
32. Schnider CM, Zabiewicz K, Holden BA. Unusual complications associated with RGP extended wear. International Contact Lens Clinic 199815 124-128.

33. Efron N. Contact lens-induced changes in corneal topography. Optician 1997 No 5626214. 